\title{
Spatial modal control of two-dimensional photonic crystal Bragg lasers
}

\author{
Lin Zhu,* Xiankai Sun, Guy A. DeRose, Axel Scherer, and Amnon Yariv \\ Department of Electrical Engineering and Department of Applied Physics, California Institute of Technology, \\ 1200 East California Boulevard, Pasadena, California 91125, USA \\ *Corresponding author: linz@caltech.edu
}

Received May 17, 2007; revised June 21, 2007; accepted June 27, 2007; posted July 6, 2007 (Doc. ID 83162); published August 2, 2007

\begin{abstract}
We investigate the modal losses and field distributions of different order transverse modes supported by the photonic crystal Bragg structure using a transfer matrix method. We find that only the fundamental transverse mode has a single-lobed near field and far field and there exists a trade-off between ensuring lasing in the fundamental transverse mode and reducing the threshold. Employing these design principles, we experimentally demonstrate a large-area, edge-emitting, and single-mode semiconductor photonic crystal Bragg laser with a single-lobed, diffraction-limited far field under continuous wave condition. (C) 2007 Optical Society of America

OCIS codes: $140.5960,130.2790,350.2770$.
\end{abstract}

Two-dimensional photonic crystal (PC) Bragg structures have been engineered to make large-area semiconductor lasers with narrow spectral linewidths and narrow beam divergences [1-9]. In our recent work, we demonstrated electrically pumped, large-area, single-mode, and edge-emitting photonic crystal Bragg lasers with angled facets operating in both pulsed and continuous wave (CW) conditions $[8,9]$. We have shown that the single-mode lasing is obtained by satisfying both the longitudinal and transverse Bragg conditions and the PC Bragg lasers are efficient for practical applications. In [9], we showed the laser oscillated in a true single mode by using an optical heterodyne method, and the laser output power was about $5 \mathrm{~mW}$ with an injected current of $70 \mathrm{~mA}$ and the slope efficiency was about $0.15 \mathrm{~W} / \mathrm{A}$. However, the far-field profile shown in [8] has two side peaks, which are detrimental for most applications since the side peaks in the far field reduce the coupling efficiency to optical fibers and planar waveguides.

In this Letter, we show that the side peaks inside the PC Bragg laser far field are associated with highorder transverse Bragg resonance (TBR) modes. We first use a transfer matrix model $[10,11]$ to compare different order transverse modes of the PC Bragg laser and find that only the first-order (fundamental) TBR mode has a single-lobed near field and thus a single-lobed far field. We show that the transverse coupling coefficient determines the intermodal discrimination between the fundamental and high-order TBR modes and there exists a trade-off between ensuring lasing in the fundamental TBR mode and reducing the threshold. We fabricate the PC Bragg lasers with different transverse coupling coefficients by changing the etch depths, and we measure the thresholds, spectra, near fields, and far fields of these lasers. Both fundamental and high-order TBR lasing modes are obtained depending on the transverse coupling coefficient. The experimental results agree well with the theoretical predictions.
Figure 1(a) shows a schematic diagram of our PC Bragg laser. The photonic crystal consists of a rectangular lattice array of polymer-filled holes each with a radius of $100 \mathrm{~nm}$ on the wafer surface. The transverse and longitudinal lattice constants are $a$ and $b$, respectively. The cleaved facet is titled relative to the transverse lattice direction by an angle $\theta_{\text {tilt }}$. We use a first-order Bragg reflection $(l=1)$ for the transverse direction with a lattice constant of $a=1 \mu \mathrm{m}$ and a second-order Bragg reflection $(j=2)$ for the longitudinal direction with a lattice constant of $b=470 \mathrm{~nm}$. The design corresponds to a resonance wavelength of $1483 \mathrm{~nm}$. (The effective index $n_{\text {eff }}$ is estimated to be 3.24.) The metal contact width is about $100 \mu \mathrm{m}$ and the facet tilt angle is $13.2^{\circ}$. Detailed fabrication procedures can be found in [8-10]. Figure 1(b) shows the cross-section structure of a fabricated PC Bragg laser.

Since we are concentrated on the modal profile in the transverse direction, we use a transfer matrix model to analyze different order transverse modes for our PC Bragg structure [11,12]. We define the modal angle as $\theta=\cos ^{-1}\left[\operatorname{Re}(\beta) /\left(2 \pi n_{\text {eff }} / \lambda\right)\right]$. Figure 2(a)

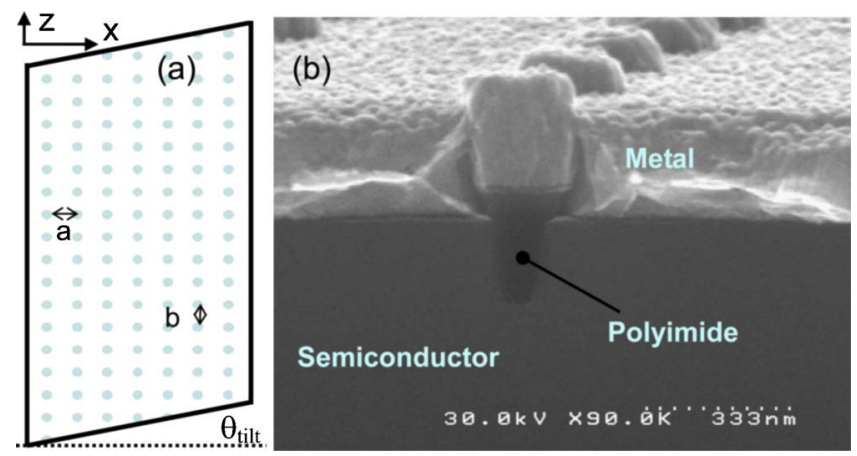

Fig. 1. (Color online) (a) Schematic of a two-dimensional photonic crystal Bragg laser. $a$ is the transverse lattice constant, $b$ is the longitudinal lattice constant, and $\theta_{t i l t}$ is the facet tilt angle. (b) The cross-section structure of a fabricated PC Bragg laser. 

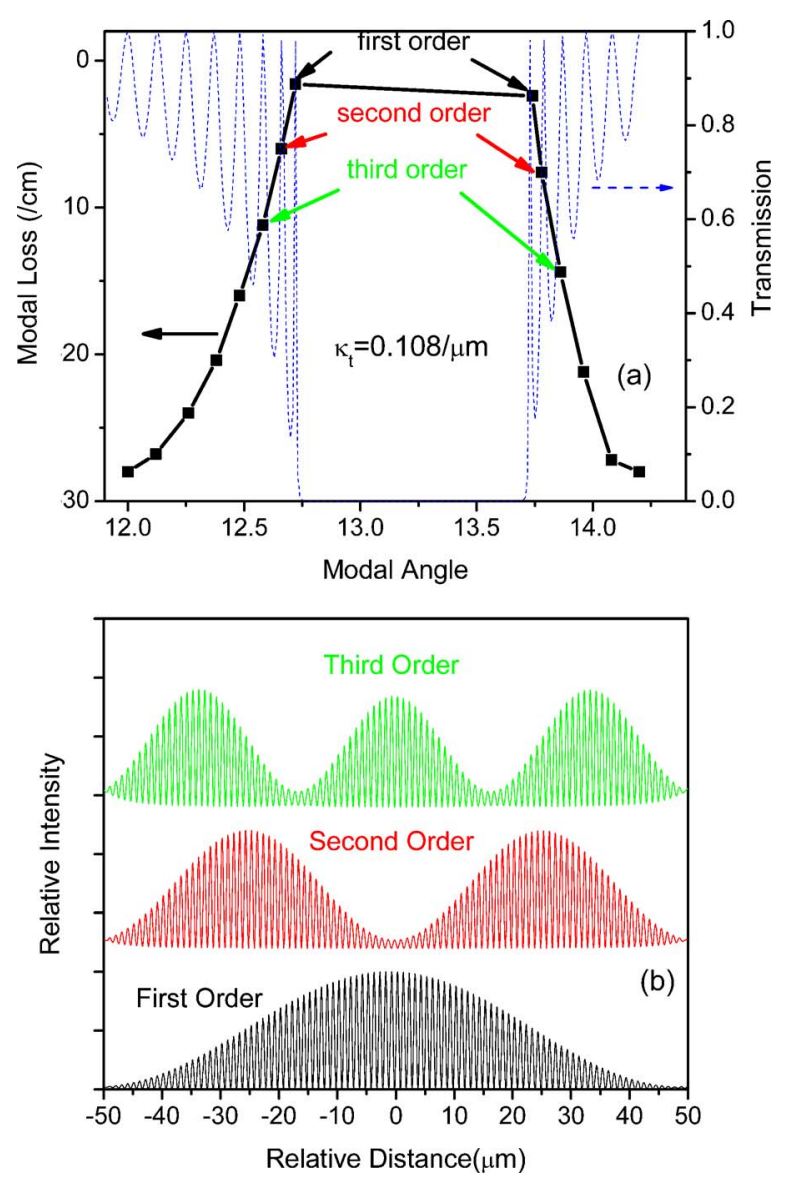

Fig. 2. (Color online) (a) Modal losses for different order TBR modes as a function of the modal angle (solid curve) and the grating transmission spectrum (dashed curve). (b) Electrical field distributions for the three lowest-order TBR modes.

shows the modal angles and modal losses for different order TBR modes, and Fig. 2(b) shows the electrical field distributions for the three lowest-order TBR modes. The transverse coupling coefficient $\kappa_{t}$, which is proportional to the index contrast [1], is $0.108 \mu \mathrm{m}$.

All the TBR modes in Fig. 2(a) are supported by the same order (first order) of Bragg reflection. In Fig. 2(a), we also calculate the transmission spectrum of the passive grating to illustrate the origin of high-order TBR modes. It is clear that each TBR mode corresponds to one peak in the grating transmission band. The finite device width (metal contact width) imposes a second transverse resonance condition and thus leads to the mode splitting. This is consistent with the fact that multiple peaks in the grating transmission band are due to the finite width of the grating. In Fig. 2(b), only the fundamental mode has a single-lobed near-field profile and thus a singlelobed far-field profile due to the angled facet design.

We need to engineer the intermodal discrimination (modal loss difference) between the fundamental and high-order TBR modes to be large enough to ensure the lasing in the fundamental TBR mode. In Fig. 3, we calculate the intermodal discrimination between the fundamental and second-order TBR modes with different transverse coupling coefficients (dashed curve).

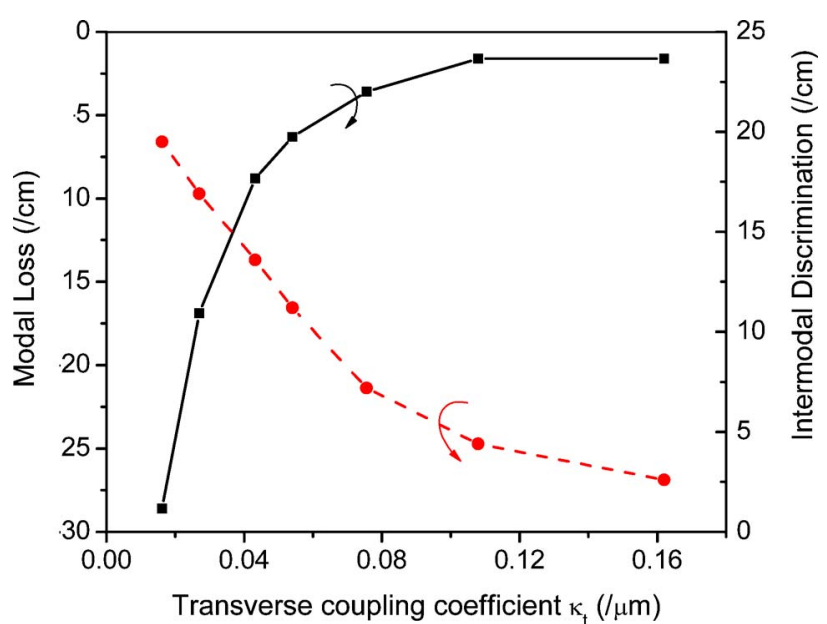

Fig. 3. (Color online) Modal losses for the fundamental TBR mode (solid curve) and intermodal discrimination between the fundamental TBR mode and second-order TBR mode (dashed curve) as a function of the transverse coupling coefficient.

In Fig. 3, it is clear that a smaller transverse coupling coefficient corresponds to a larger modal discrimination against high-order TBR modes. However, the modal loss for the fundamental TBR mode increases as the transverse coupling coefficient decreases, since the grating reflectivity becomes smaller. Thus, there exists a trade-off between obtaining the single-lobed, diffraction-limited far field (ensuring the lasing in the fundamental mode) and reducing the threshold, for a given device width. To illustrate this important design issue, we also calculate the modal loss of the fundamental TBR mode as a function of the transverse coupling coefficient in Fig. 3 (solid curve). From Fig. 3, we expect the optimum transverse coupling coefficient in the range of $0.02 \mu \mathrm{m}^{-1}<\kappa_{t}<0.08 \mu \mathrm{m}^{-1}$ to obtain the lasing in the fundamental mode combined with a relatively low threshold. Since the modal waveguiding loss is only one of a number of comparable loss mechanisms such as scattering, absorption, and output coupling, which in total are comparable in magnitude to the modal loss, we expect a relatively small variation of the laser threshold over the range of $0.02 \mu \mathrm{m}<\kappa_{t}$ $<0.08 \mu \mathrm{m}$.

Relying on the theoretical analysis, we fabricate the PC Bragg lasers with three different etch depths of 250,305 , and $430 \mathrm{~nm}$. The corresponding transverse coupling coefficients are $0.012,0.024$, and $0.122 \mu \mathrm{m}$, respectively. These lasers are tested at $150 \mathrm{~K}$ to obtain the $\mathrm{CW}$ lasing. The laser with etch depth of $250 \mathrm{~nm}$ does not lase due to the high modal loss (we injected the current up to $300 \mathrm{~mA}$ ). The threshold currents for the lasers with etch depths of 305 and $430 \mathrm{~nm}$ are 120 and $58 \mathrm{~mA}$, respectively. The lasers have similar performance as we reported in [9] in terms of the slope efficiency, output power, and single modeness. The measurement results of the spectra, near fields, and far fields for these two lasers are shown in Fig. 4.

Single-mode lasing is obtained for both lasers with more than $30 \mathrm{~dB}$ side mode suppression ratios, as 

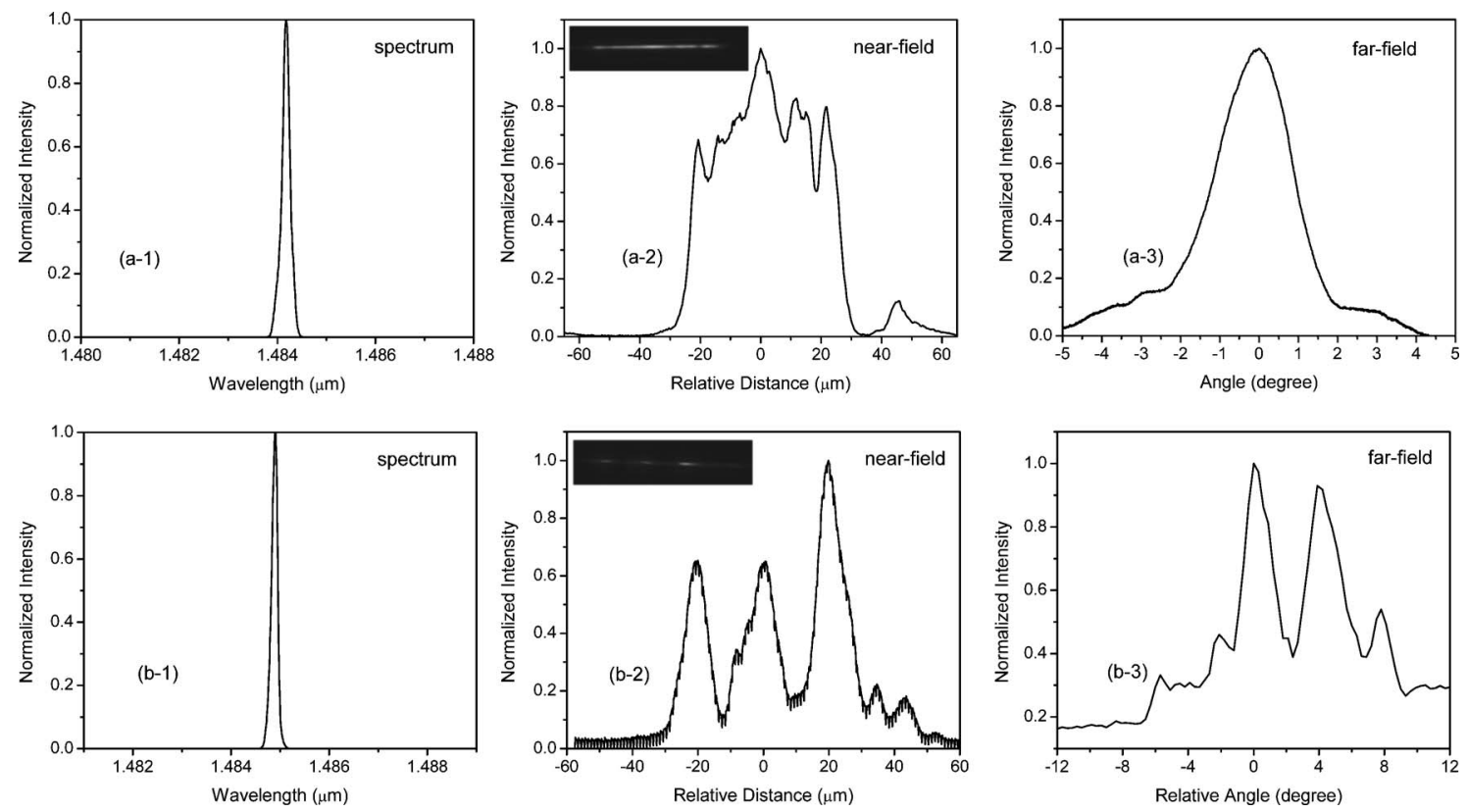

Fig. 4. Measurement results of the PC Bragg lasers with different etch depths. All the measurements are taken at $I$ = 1.6 ith. (a.1)-(a.3) Spectrum, near field, and far field of the PC Bragg laser with 305 nm etch depth. (b.1)-(b.3) Spectrum, near field, and far field of the PC Bragg laser with $430 \mathrm{~nm}$ etch depth. The insets in (a-2) and (b-2) are direct infrared images of the laser facets.

shown in Figs. 4(a-1) and 4(b-1). In Figs. 4(a-2) and 4(a-3), the laser with $305 \mathrm{~nm}$ etch depth has a singlelobed near field and a single-lobed far field. The FWHM width of the near-field profile is about $50 \mu \mathrm{m}$, corresponding to a theoretical diffraction-limited farfield FWHM width of $1.97^{\circ}$ [13]. The measured FWHM width of the laser far-field profile is about $2^{\circ}$, which proves that single-lobed, diffraction-limited far field is obtained for this single-mode laser. In Figs. $4(\mathrm{~b}-2)$ and 4(b-3), the near field and far field of the laser with $430 \mathrm{~nm}$ etch depth both have three lobes, which corresponds to the lasing in the third order TBR mode.

It should be pointed out that our theoretical calculations assume a uniform material gain (loss) distribution. Thus, the fundamental mode always has a lower threshold compared with high-order modes. However in the fabricated devices, if the transverse coupling coefficient is large, high-order modes may possess a lower threshold depending on the actual gain distribution. This explains why the single-mode lasing in the third-order TBR mode is obtained for the device shown in Fig. 4(b).

In summary, we use a transfer matrix approach to calculate the modal losses of different order TBR modes and compare their field distributions. Our theoretical calculations show that there exists a tradeoff between obtaining lasing in the fundamental TBR mode with a single-lobed, diffraction-limited far field and reducing the threshold. Guided by the theoretical analysis, we obtain the single-mode lasing of the PC Bragg laser with a single-lobe, diffraction-limited far field by optimizing the transverse coupling coefficient of the photonic crystal lattice.

L. Zhu thanks O. Painter and R. Perahia for access to their PECVD facility. J. Poon is thanked for fruit- ful discussions. The work was supported by the Airforce Office of Scientific Research (H. Schlossberg) and by DARPA (J. Mangano). This work was also supported by a subaward from the Office of Naval Research under contract N00014-05-M-0254.

\section{References}

1. A. Yariv, Opt. Lett. 27, 936 (2002).

2. I. Vurgaftman and J. R. Meyer, IEEE J. Quantum Electron. 38, 592 (2002).

3. C. S. Kim, W. W. Bewley, C. L. Canedy, I. Vurgaftman, M. Kim, and J. R. Meyer, IEEE Photon. Technol. Lett. 16, 1250 (2004).

4. S. Noda, M. Yokoyama, M. Imada, A. Chutinan, and M. Mochizuki, Science 293, 1123 (2001).

5. D. Ohnishi, T. Okano, M. Imada, and S. Noda, Opt. Express 12, 1562 (2004).

6. L. Zhu, J. M. Choi, G. A. DeRose, A. Yariv, and A. Scherer, Opt. Lett. 31, 1863 (2006).

7. H. Hofmann, H. Scherer, S. Deubert, M. Kamp, and A. Forchel, Appl. Phys. Lett. 90, 121 (2007).

8. L. Zhu, G. A. DeRose, A. Scherer, and A. Yariv, Opt. Lett. 32, 1256 (2007).

9. L. Zhu, X. K. Sun, G. A. DeRose, A. Scherer, and A. Yariv, Appl. Phys. Lett. 90, 26116 (2007).

10. L. Zhu, P. Chak, J. K. S. Poon, G. A. DeRose, A. Scherer, and A. Yariv, Opt. Express 15, 5966 (2007).

11. R. J. Lang, K. D. Zurko, A. Hardy, S. Demars, A. Schoenfelder, and D. Welch, IEEE J. Quantum Electron. 34, 2196 (1998).

12. L. Zhu, A. Yariv, and A. Scherer, "Modal gain analysis of transverse Bragg resonance waveguide lasers with and without transverse defects" IEEE J. Quantum Electron. (to be published).

13. D. Botez and D. R. Scifres, Diode Laser Arrays (Cambridge U. Press, 1986), p. 12. 